\title{
Combining geometric edge detectors for feature detection
}

\author{
Michaël Heyvaert, David Van Hamme, Jonas Coppens, Peter Veelaert \\ University College Ghent, Engineering Sciences - Ghent University Association, \\ Schoonmeersstraat 52, B9000 Ghent, Belgium \\ michael.heyvaert@hogent.be david.vanhamme@hogent.be \\ jonas.coppens@hogent.be peter.veelaertahogent.be
}

\begin{abstract}
We propose a novel framework for the analysis and modeling of discrete edge filters, based on the notion of signed rays. This framework will allow us to easily deduce the geometric and localization properties of a family of firstorder filters, and use this information to design custom filter banks for specific applications. As an example, a set of angle-selective corner detectors is constructed for the detection of buildings in video sequences. This clearly illustrates the merit of the theory for solving practical recognition problems.
\end{abstract}

\section{Introduction}

The traditional approach in feature detection is to start with filter kernels that yield accurate localization in space and frequency [12]. General corner detectors, e.g. Harris, FAST and SUSAN are rotationally insensitive, as this is a desirable property in many feature matching applications. Steerable filters, e.g. Gabor filters and Derivative of Gaussian (DoG) filters, incorporate orientation to increase the selectivity. Since one cannot convolve an image with kernels for all possible orientations, scales and positions, only a small set of filters is used to sample the space for a finite set of orientations and scales. The response for arbitrary orientations or scales is obtained by interpolation.

The detection of more specific patterns, such as wedges or junctions, requires specially tuned filters. Perona demonstrates the use of 2-sided, endstopped and 1-sided endstopped filters for the detection of junctions, where each of these tuned filters is a suitable combination of a limited set of basic kernels [12]. Simoncelli et al use steerable wedge filters to avoid 180 degree periodic response [11]. Jacob et al use the degrees of freedom of steerable functions to design shape-adaptable filters, in particular for ridges and wedges [4]. The drawback of using steerable filters, however, is that basic kernels of high order are needed, requiring relatively large templates (typically with radius 10 or larger).

In this work we construct filters in a design space which differs from the classical approach. The major novelty is that we will exploit the relation between position selectivity and orientation selectivity. We will make explicit use of the property that the orientation selectivity actually depends on the position of the edge relative to the filter kernel. To highlight the advantages of the proposed scheme we will use filter response models that are relatively simple, without referring to scale or frequency. 
Modeling the dependency between position uncertainty and orientation uncertainty makes it easier to design detectors for specific features. To detect features that consist of two or more edge segments, e.g., corners or junctions, we have to combine the response of multiple edge detectors for edges at different orientations and positions. The proposed scheme indicates which edge detectors have to be combined. Furthermore, there is still the freedom to choose the selectivity of the edge filters that will be used. The inclusion of a large number of highly selective filters will yield high accuracy. A small set of filters with limited selectivity requires less computation. The edge filters used in this work all have a small radius $(\leq 2.5)$ with very few non-zero coefficients. The filters are much smaller and simpler than the high-order steerable filters, since we combine filter responses at different positions in the image.

Section 2 introduces the mathematical model used to characterize edge filters. Section 3 shows how to evaluate the performance of combined edge filters. The technique is illustrated in Section 4 for the detection of corners in buildings.

\section{Discrete geometry approach to edge detection}

To model the dependency between orientation and position selectivity we need to define some concepts related to the digitization of straight edges and lines.

Let $U=\left\{u_{1}, \ldots, u_{n}\right\}$ and $V=\left\{v_{1}, \ldots, v_{m}\right\}$ be two finite and non-empty subsets of $Z^{2}$ such that $U$ can be linearly separated from $V$. Then the lines separating these regions satisfy the following conditions:

$$
\begin{gathered}
y_{i}-a x_{i}-b>0,\left(x_{i}, y_{i}\right) \in U \\
y_{j}-a x_{j}-b<0,\left(x_{j}, y_{j}\right) \in V
\end{gathered}
$$

Thus the parameters of the separating lines lie in an open convex region or domain in the $a b$-parameter plane, which will be denoted as $D(U, V)$. This domain is related to the preimage of a discrete straight line segment [5], but it is not exactly the same concept, since the preimage of a discrete subset considers all the lines that stab a set of pixel squares, while here we consider the lines that separate two sets of points.

Fig. 1(a) shows two disjoint point sets. The straight lines that separate these two sets cover a butterfly shaped region. We note that the range of possible orientations of lines that pass through $p_{1}$ is different from the range of orientations at the point $p_{2}$. Likewise, given an arbitrary straight line which separates $U$ and $V$, the distance by which this line can be displaced while keeping the separation intact depends on the orientation of the line. For example, the line $L_{1}$ can be displaced much further vertically than the line $L_{2}$. Also in Fig. 1(b) it is clear that there is a relation between position and slope uncertainty. Fig. 1(b) shows the domain that contains the parameters of the separating lines. When the domain is sliced into vertical segments, the height of each vertical segment depends on the position of the segment in the domain, that is, on the slope parameter $a$.

The digital edge filters used in this work are first order filters that are directly related to two disjoint sets of grid points. Let $f(x, y): R^{2} \rightarrow R$ be an image function of two continuous variables. We use a simple sampling model: a digital image $f[x, y]$ is formed from the function $f(x, y)$ by the relationship $f[x, y]=f(x, y)$, for integer values of $x$ and $y, 0 \leq x \leq M, 0 \leq y \leq N$. 


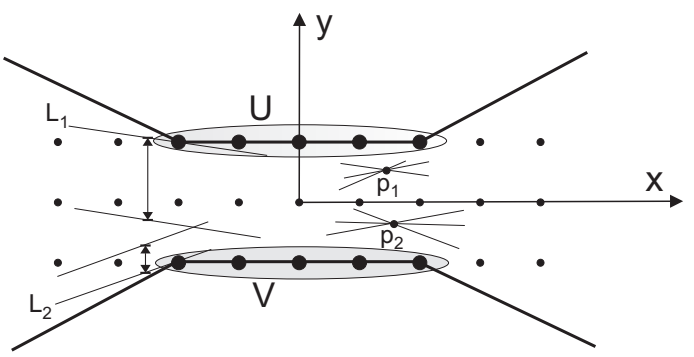

(a)

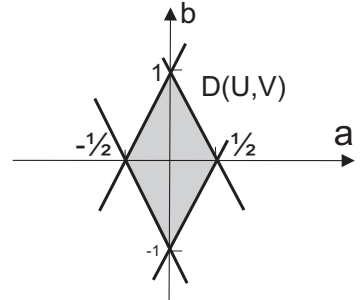

(b)

Fig. 1. (a) Straight lines that separate two discrete sets $U$ and $V$, and (b) the parameter domain of all the separating lines.

Let $U=\left\{u_{1}, \ldots, u_{n}\right\}$ and $V=\left\{v_{1}, \ldots, v_{m}\right\}$ be two disjoint subsets of $Z^{2}$. We define a digital 2D filter $h[x, y]$ as follows:

$$
h[x, y]= \begin{cases}\frac{1}{2 n} & \mid(x, y) \in U \\ \frac{-1}{2 m} & \mid(x, y) \in V \\ 0 & \text { otherwise }\end{cases}
$$

When we apply the filter $h[x, y]$ to the digital image of an edge, i.e. $g=h * u$, and consider its response at $g[0,0]$, we find that there is direct relation between the filter response and the domain $D(U, V)$. Let $u(x, y)$ denote an edge function of the form $u(x, y)=1$ if $0 \leq y-a x-b$, and $u(x, y)=-1$ if $0>y-a x-b$, for some real numbers $a, b$. Let $u[x, y]$ denote the discrete sampling of $u(x, y)$. Let $g[]=h[] * u[]$ denote the response of the filter for the edge function $u[x, y]$. Clearly, the response $|g[0,0]|$ will be maximal, i.e, $|g[0,0]|=1$ provided $u[x, y]=1$ for all $(x, y) \in U$ and $u[x, y]=-1$ for all $(x, y) \in V$. The values of $a$ and $b$ for which this holds true define the edges that separate the two sets $U$ and $V$. Their line parameters $a, b$ lie in the domain shown in Fig. 1. For any other edge outside the domain the response $|g[0,0]|$ will be less than 1 . To summarize, the filter response is maximal for the edges whose parameters lie in the domain $D(U, V)$.
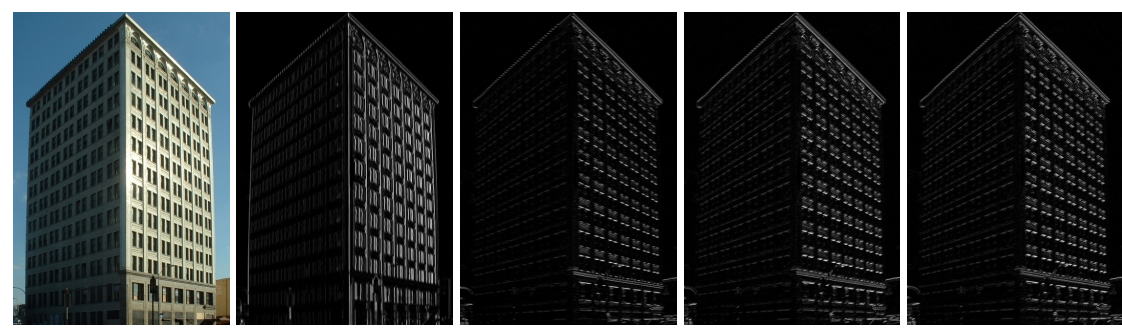

Fig. 2. Response of four filters with different orientations. 


\subsection{Signed rays}

To be able to combine filters for the detection of features we will decompose domains into elementary domain cells. To this end we introduce the simple but convenient notion of a signed ray of points. A signed set $X$ is a set together with a partition $\left\{X_{p}, X_{n}\right\}$ where the elements in $X_{p}$ have positive signs, and the elements in $X_{n}$ have negative signs.

Definition 1. Let $S$ be a finite set of points in the plane, and let $R$ be an ordered signed subset of $S$. Then $R$ is a signed ray if:

- $R$ is the intersection of a straight line with $S$;

- the ordering of $R$ preserves the betweenness-relation of Euclidean geometry;

- the sign changes only once along the ray.

A signed ray can be denoted as $R=\ldots p_{1} p_{2} \overline{p_{3} p_{4}} \ldots$, where $p_{1}$ and $p_{2}$ have positive signs, and $p_{3}$ and $p_{4}$ have negative signs. Because of the last condition in the definition, we cannot have rays of the form $p_{1} p_{2} \overline{p_{3} p_{4}} p_{5} \ldots$, where the sign alternates more than once. But it is possible to start with negative signs, e.g., $R=\overline{p_{1} p_{2}} p_{3} p_{4}$. All points may also have the same sign.

A signed ray $R=p_{1} \ldots \overline{p_{n}}$ divides the points of $S$ into two parts. The points in $R \cap S$ already have a sign. Let $L$ be the straight line passing through the points of $R$. Since $R$ is an ordered sequence, the points induce an orientation on the line $L$, from $p_{1}$ to $p_{n}$. With this orientation we can attribute signs to the points in $S$ not in $R$. Points that lie at the right side of $L$ receive a positive sign, points at the left side receive a negative sign. The two signed parts of $S$ will be denoted as $R^{+}$and $R^{-}$, respectively. Thus the signs of the points of the ray serve to add these points to either $R^{+}$or $R^{-}$.

There is a direct relation between rays and linear separations of sets.

Proposition 1. Let $S=S^{+} \cup S^{-}$be a partition of a finite set $S$ induced by a straight line $L$ that does not pass through any of the points of $S$. Then there exists a signed ray $R$ such that $S^{+}=R^{+}$and $S^{-}=R^{-}$. Conversely, if $R$ is a signed ray in $S$, then the subsets $R^{+}$and $R^{-}$can be linearly separated by a straight line not passing through any of the points of $S$.

As a result each signed ray corresponds to a domain. Suppose $R$ separates the points of $S$ into two non-empty parts, $S^{+}$and $S^{-}$. The straight lines separating $S^{+}$and $S^{-}$ satisfy the following conditions:

$$
\begin{gathered}
y_{i}-a x_{i}-b>0,\left(x_{i}, y_{i}\right) \in S^{+} \\
y_{j}-a x_{j}-b<0,\left(x_{j}, y_{j}\right) \in S^{-}
\end{gathered}
$$

The parameters $a$ and $b$ that satisfy these conditions occupy an open convex region in parameter space, the domain of the signed ray, which is denoted as $D_{S}\left(R^{+}, R^{-}\right)$.

Distinct rays may represent the same domain. Fig. 3 shows four distinct rays that separate the four points such that $p_{1}, p_{2}$ receive positive signs and $p_{3}, p_{4}$ negative signs, namely, $p_{1} p_{2}, \overline{p_{3}} p_{2}, p_{1} \overline{p_{4}}$, and $\overline{p_{3} p_{4}}$. Likewise, $\overline{p_{2} p_{1}}, \overline{p_{2}} p_{3}, p_{4} \overline{p_{1}}$, and $p_{4} p_{3}$ separate the points such that $p_{1}, p_{2}$ receive negative signs and $p_{3}, p_{4}$ positive signs, which yields the 
same partition but with interchanged signs. Among these 8 rays of the same partition, we can select a unique ray by imposing an ordering relation on the points of $S$ (not to be confused with the ordering of $R$ ). For example, with $p_{1}<p_{2}<\ldots<p_{n}$, and using lexicographic ordering, the smallest representant is $p_{1} p_{2}$. From now on, we will use the term 'signed ray' to denote the smallest representant corresponding to a certain partition of $S$.

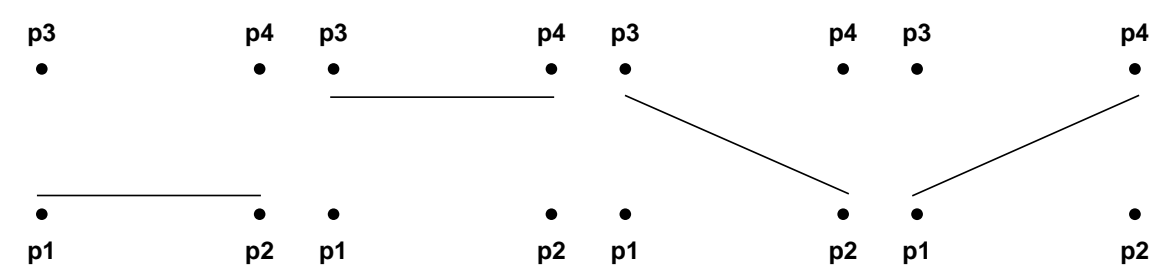

Fig. 3. Four signed rays that separate the points into $\left\{p_{1}, p_{2}\right\}$ and $\left\{p_{3}, p_{4}\right\}:$ (a) $p_{1} p_{2}$, (b) $\overline{p_{3} p_{4}}$, (c) $\overline{p_{3}} p_{2}$, and (d) $p_{1} \overline{p_{4}}$

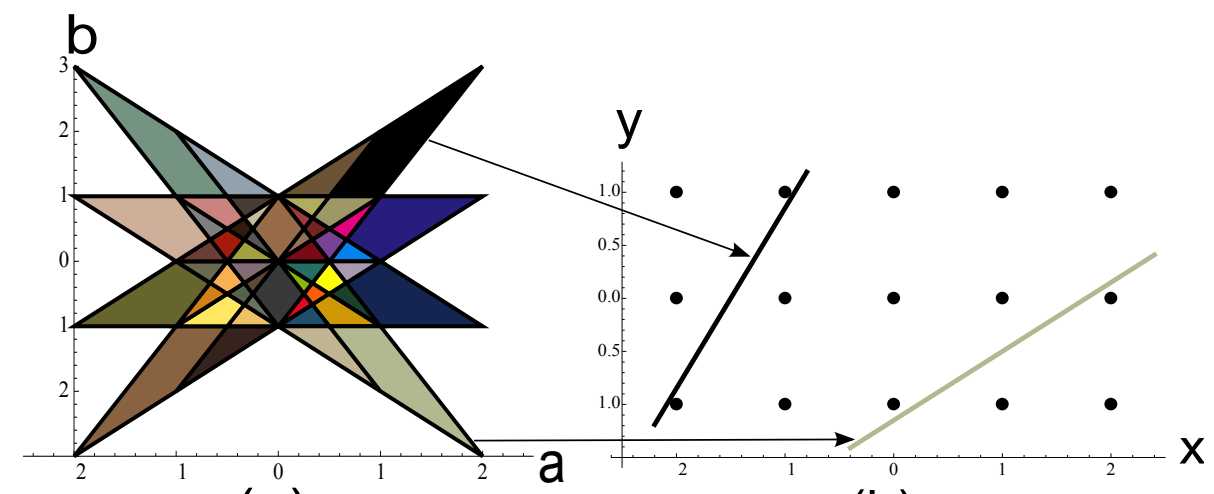

(a)

(b)

Fig. 4. (a) Finite domains of the signed rays for a $3 \times 5$ set of points. (b) Examples of member lines for two of the domains.

Fig. 4(a) shows the bounded domains for the set $S$ of $3 \times 5$ grid points shown in Fig. 4(b). Only domains that are bounded are shown, i.e, domains that do not include parameters of vertical lines.

With rays we decompose domains into elementary domains. Let $U$ and $V$ be subsets of a set $S$, such that $U$ and $V$ can be linearly separated. The union $U \cup V$ does not necessarily constitute the entire set $S$. Clearly the domain of lines that separate $U$ and $V$ can be decomposed into elementary domains that correspond to rays. Let $R(U, V)$ 
denote the set of all signed rays $R$ that divide the set $S$ into parts $R^{+}$and $R^{-}$so that either $V \subseteq R^{+} \wedge U \subseteq R^{-}$or $U \subseteq R^{+} \wedge V \subseteq R^{-}$. The topological closure of the domain of the disjoint subsets is the union of the closure of the elementary domains of the rays in $R(U, V)$ :

$$
\operatorname{cl}(D(U, V))=\cup_{R \in R(U, V)} \operatorname{cl}\left(D_{S}\left(R^{+}, R^{-}\right)\right)
$$

In fact, $R(U, V)$ corresponds to all the possible ways in which the subsets $U$ and $V$ can be maximally extended until their extensions coincide with a complete linear separation of $S$. Furthermore, no pair of subsets in $S$ can have a domain $D(U, V)$ smaller than that of any ray in $S$. and the domains of two distinct rays are always disjoint.

The size of the set $S$ defines the resolution of the decomposition into elementary domains. When $S$ is enlarged, the ray domains become smaller, that is, if $S_{1} \subseteq S_{2}$ then $\operatorname{cl} D_{S_{1}}\left(R^{+}, R^{-}\right) \supseteq \operatorname{cl} D_{S_{2}}\left(R^{+}, R^{-}\right)$.

Ray domains are very similar to the cells in a Farey fan for digitized straight lines as defined in $[2,5]$. The definition of a ray domain is more general, however, as the decomposition can be defined for an arbitrary set $S$. Furthermore, as mentioned earlier, ray domains correspond to sets of separating lines, while Farey cells correspond to common line stabbings.

The importance of decomposing domains of disjoint subsets is that a first order edge filter can be considered as the detector of a given collection of partitions of $S$. The decomposition into elementary domains, where each elementary domain corresponds to a filter with maximal selectivity, therefore allows us to identify all the possible overlaps and gaps in a bank of edge filters.

\subsection{Localization constraints}

A second reason for introducing rays is that they are well suited to formulate constraints concerning localization, e.g. all straight lines passing through a polygon. Let us assume that the vertices of the polygon are points of $S$. In practical applications, this is a reasonable assumption, since it only requires that the vertices coincide with image sample points. Then it is sufficient to list all rays that do not attribute the same sign to all vertices. To be explicit, let $P$ be a set of vertices of a convex polygon, with $P \subset S$, then the closed set

$$
\cup_{R \mid R^{+} \cap P \neq \emptyset \wedge R^{-} \cap P \neq \emptyset} \mathrm{cl} D_{S}\left(R^{+}, R^{-}\right)
$$

contains the parameters of the straight lines that pass through the polygon $P$.

If the vertices of the polygon are not points of $S$, the use of rays can still hold merit. Consider the extension of this polygon to its smallest bounding polygon made up by points in $S$. Any ray that does not cross this bounding polygon, cannot contain a line which crosses the original polygon. Thus rays can still be used to form boundaries delimiting the parameter space to be searched.

\subsection{Angular relations}

Rays were introduced to discretize the parameter space of straight lines. As a result, angles between rays are determined in terms of intervals. For each pair of rays we can 
determine the minimum and maximum angle between any two of their lines by looking at the extrema of their domains in the $a$-axis (see Fig. 1). Rays are considered parallel when the angle intervals overlap, that is, when their domains contain at least one pair of parallel lines. Similarly, two rays are considered perpendicular if their domains contain at least one perpendicular pair of lines.

\section{Combining edge detectors}

The well-defined geometric properties and localization constraints of rays and the domain based approach allow us to easily design custom detectors for compound structures such as perpendicular corners, T-junctions or Y-junctions. Each detector will consist of one or more simple filters, and in general, multiple detectors will be needed to detect all occurrences of a feature. Furthermore, the size and the shape of the filters will determine their selectivity. The performance of each detector can be evaluated by computing ray domains. The general approach is as follows:

- First we select a set $S$ of grid points for which all the ray domains will be computed. The set $S$ determines the resolution of the domains. It makes no sense to choose $S$ too large, however. It suffices that $S$ encompasses all the sample points of the digital filters that will be used, and the vertices of the polygons that are used to define localization constraints.

- Next, we list all the rays or combinations of rays that define the features that we are interested in. The result is a list of ray tuples, for example pairs of rays that form corners, or triples of rays that form $Y$-junctions.

- Feature detectors are constructed by combining edge detectors. For each feature detector we can verify which ray tuples are detected, i.e, for which the detector will yield a maximal response. This will show whether all tuples are covered. The selectivity of the detectors is examined by looking at ray tuples for which more than one detector responds or by verifying whether the detector responds to tuples that do not correspond to the features that we are interested in.

Fig. 5 illustrates a simple example. The features that we are interested consist of perpendicular corners, for which one edge has a slope in $[0.85 ; 1.15]$ and the second edge has a slope in $[-1.15 ;-0.85]$. To make sure that we can detect this feature at any position in the image, we will examine the detection performance in the gray square shown in Fig. 5. The vertices of the square are four neighboring pixels. The detector that will be analyzed consist of the two simple filters $F$ and $G$ shown in Fig. 5, with filter coefficients equal to $-1 / 2$ at the dark dots, and equal to $1 / 3$ at the light dots.

Fig. 6(a) shows the domains of all rays that contain lines passing through the square and have one of the required slopes. In this case a domain is included as soon as its closure contains a line with a slope in the specified intervals. Since there are 14 domains at the left side and 14 domains at the right side we can form 196 ray pairs. To select perpendicular corners, in this collection of 196 pairs all the ray pairs are listed that contain at least one perpendicular pair of lines. Fig. 6(b) shows a domain $D$ that contains lines with slope in $[0.85 ; 1.15]$. The domains at the left side of Fig. 6(b) each contain at 


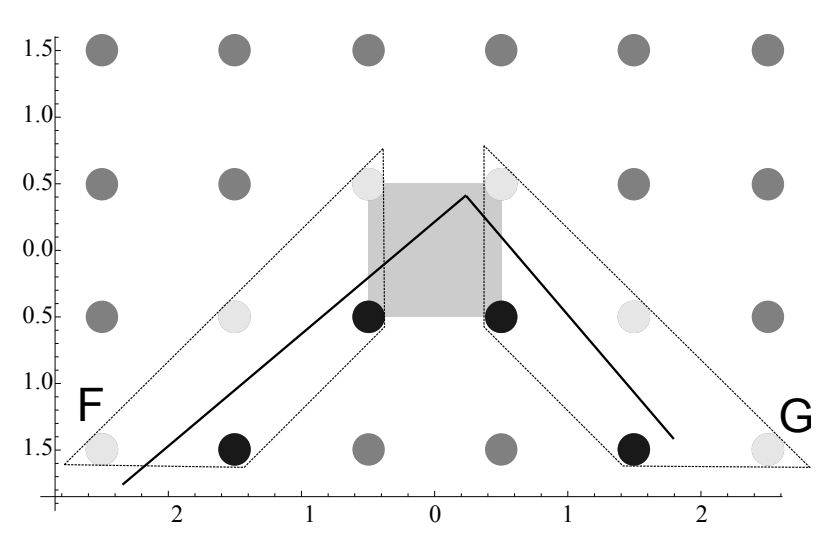

Fig. 5. Detection of perpendicular corners in a square. Two filters $F$ and $G$ are shown.

least one line that is perpendicular to one of the lines in $D$. In total there are 122 ray pairs that contain perpendicular corners.

Fig. 6(c) shows to which ray pairs the filters $F$ and $G$ will both give a maximal response. At the left side of Fig. 6(c) the ray domains are shown for which $G$ responds, at the right side those for which $F$ responds. Note that $F$ (and $G$ ) also responds to a domain with lines that do not have the required slope. Since each filter gives a maximal response for 5 domains, the detector formed by $F$ and $G$ is triggered by 25 distinct ray pairs. Only 13 of them, however, contain orthogonal corners. Hence, the detector is not very selective since about half of the pairs by which it is triggered do not contain perpendicular corners. Clearly, the selectivity of the detector can be improved by using filters with a larger extent defined in a larger set $S$. Furthermore, the filter pair $F, G$ only covers 13 of the 122 perpendicular pairs. More corner pairs can be covered by including more filter pairs, as will be illustrated in the next section.

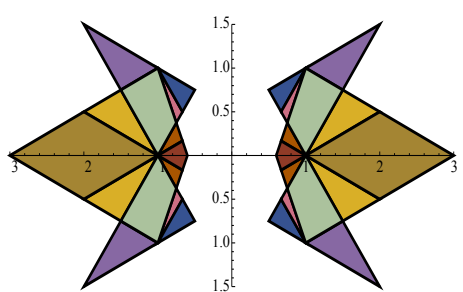

(a)

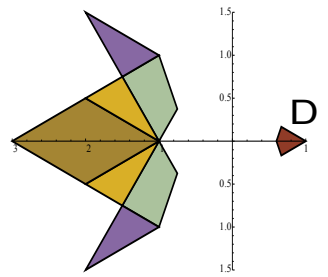

(b)

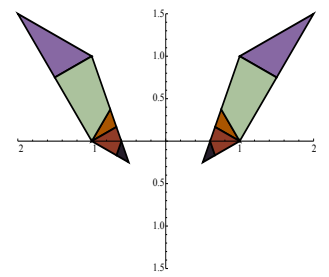

(c)

Fig. 6. Domains of the signed rays for a $4 \times 6$ set of points. (a) Shows all the domains of rays that cover slopes of either 1 or -1 and that enter the gray square. (b) Orthogonal pairs of domains. (c) Domain pairs for which both $F$ and $G$ yield a maximal response.

Also note that although Fig. 6(a) only considers rays that cross the square, the detector may respond to pairs of perpendicular lines that intersect outside the square. This 


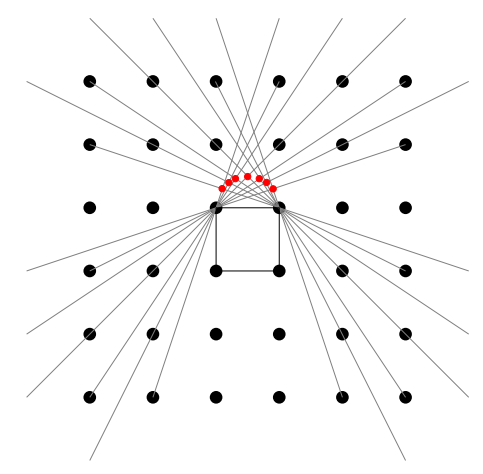

Fig. 7. Illustration of outer bound for intersection of perpendicular lines crossing a square.

localization inaccuracy is bounded, however, by four half-circles with a side of the square as diameter, and is therefore also independent of the size of the grid $S$ in which the rays are considered. This is illustrated in Fig. 7.

\section{Application: corner detection in buildings}

One of the most defining features of buildings and man-made structures in general is that they tend to be constructed almost exclusively out of horizontal and vertical surfaces. The presence of a multitude of perpendicular corners is therefore a useful feature for detecting buildings in video. However, as they are viewed through a perspective transformation, these corners can be significantly skewed. In common cinematography, two of the camera axes are more or less perpendicular to the ground plane. This means that the vertical edge of a perpendicular corner can be assumed to remain vertical through the perspective transformation, while the horizontal edge will generally be rotated. We therefore need a set of detectors which respond maximally to any combination of a vertical with a non-vertical edge.

We will construct a filter bank following the reasoning of the previous section. This means combining a first order filter which responds maximally to vertical rays crossing a square, with a complete set of first order filters which respond maximally to all other orientations of rays crossing the square. Note that the minimum angle of the corner is defined by the orientation selectivity of the vertical first order filter, which itself is bounded by the grid size in which it is defined.

As a proof of concept we design a bank of filters as shown in Fig. 8. Only the filters for rays between 0 and 45 degrees are pictured. The remainder can be obtained by mirroring over the horizontal and vertical axes, resulting in a bank of 52 unique filters. For implementation purposes, further reduction is possible, as many of the filters have an identical point configuration, but a different point of origin.

By enumerating for each filter the rays to which responds, we can prove that this set of filters is both complete and non-overlapping for a 2 by 2 square, meaning that for each line which crosses a 2 by 2 square there is exactly one filter which yields maximal 


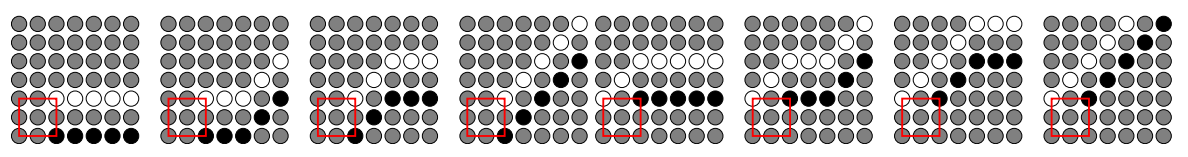

Fig. 8. 8 first order filters from a bank of 52. The remainder is obtained through horizontal and vertical mirroring.

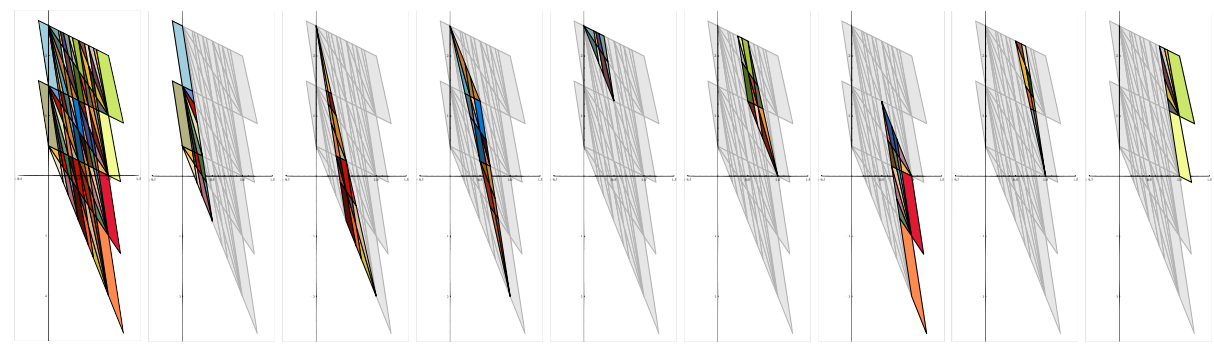

Fig. 9. Domains in the $a b$ parameter space of all rays containing lines crossing the 2 by 2 square with a slope between 0 and 1 (left), and of rays with maximal response for each of the filters (remainder).

response. The proof is illustrated in Fig. 9. The leftmost graph shows the parameter domains of all rays in the 7 by 7 array containing a line angled between 0 and 45 degrees which crosses the 2 by 2 square. The remaining graphs show the parameter domains of maximal response for each filter shown in Fig. 8. In Fig. 9 it can be seen that the union of the domains of the individual filters makes up the whole set of domains as shown on the left, while the intersection between the filters is empty. Due to the symmetries, these properties apply to the entire filter set.

To translate the theoretical results to a practical application, we need to swap the notion of maximal response with a threshold on the response, as real edges in an image do not correspond to the theoretical concept of the infinitely sharp edge. In this case, a locally adaptive threshold was chosen based on the average intensity of the image patch with a Gaussian filter mask of size $11 \times 11$. Next each non-vertical response is thresholded and combined with the vertical responses. The resulting masks contain all the detected corners. Due to the non-ideal edges, a non-maximal suppression step is needed to remove multiple detections of the same corner. This step is implemented by only retaining the corner with the strongest response in a $3 \times 3$ neighborhood. The measure for determining the strongest response is the sum of the squared response magnitudes for both edges.

Fig. 10 shows corners angle indicated with the different color labels. The angular selectivity of the filters divides the building into zones of similar perspective skew. This is a good illustration of the orientation selectivity of the filter pairs. We also compared the detected corners with the Harris corner detector [3]. Fig. 11 shows the 400 strongest Harris corners, and the 400 strongest corners according to our method. The results of 

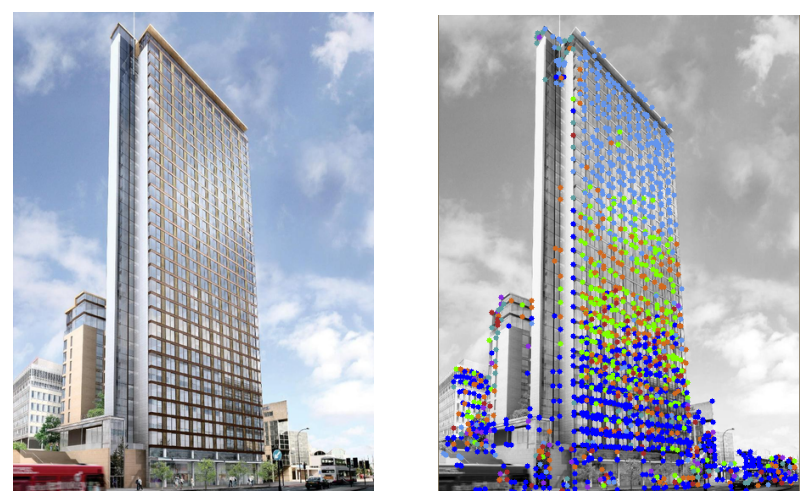

Fig. 10. Corner detection, the color gives a coarse indication of the corner angle.

the theoretical domain analysis are confirmed in several ways. First, when both methods detect the same corner, the positions coincide, proving the accuracy of localization. Second, our corners cover $48 \%$ of the Harris corner set, which is not surprising, as the detectors were designed for a limited set of corner shapes, while Harris is a general corner detector. On the other hand, our method provides a classification of the corners into corner types.

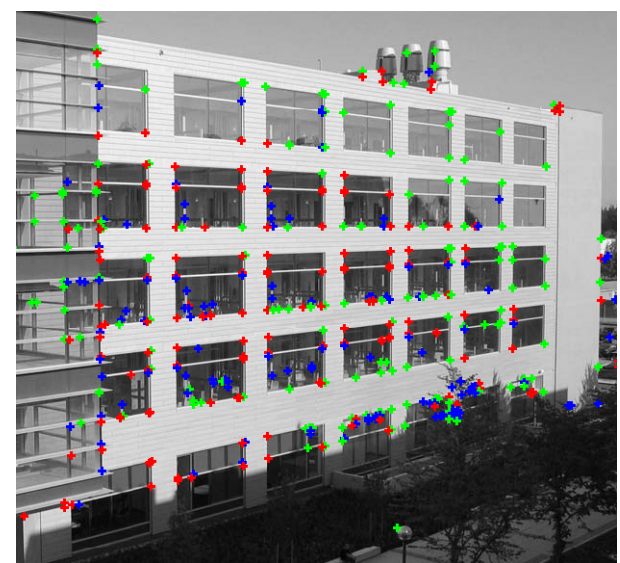

Fig. 11. Comparison of our corner detector and the Harris corner detector, red corners are matches, green corners are only detected by our method and blue corners are only detected by the Harris corner detector. 


\section{Conclusion}

We have proposed a theoretical framework to model the properties of first-order filters in a discrete geometric approach. The definition of rays allows us to simply deduce localization and geometric properties of families of filters, and offers an interesting insight into the consequences of a discrete sampling of edges in a continuous image function. The usefulness of the theory is illustrated by an example application, where the angular properties of real-world structures are evaluated by a custom filter set. Some aspects remain to be explored in more depth, such as the relation between angular selectivity and choice of grid size and density, and the best way to construct first order filters from the chosen grid. Even so, we can conclude that the ray-based framework is already a useful tool to model the properties of discrete filters.

\section{References}

1. Chinneck, J.: Feasibility and Infeasibility in Optimization. International Series in Operations Research and Management Science, Springer. (2008)

2. Coeurjolly, D., Sivignon, I.: Measure of straight lines and its applications in digital geometry. Progress in Combinatorial Image Analysis, Research Publishing (2010) 37-48

3. Harris, C., Stephens, M.: A combined corner and edge detector. In: Alvey Vision Conference. (1988) 147-151

4. Jacob, M., Unser, M.: Design of Steerable Filters for Feature Detection Using Canny-Like Criteria. IEEE Transactions on Pattern Analysis and Machine Intelligence, 26 (2004) 10071019

5. Lindenbaum, M., Bruckstein, A.: On recursive $O(N)$ partitioning of a digitized curve into digital straight line segments. IEEE Transactions on Pattern Analysis and Machine Intelligence, 15 (1993) 949-953

6. Lowe, D.G.: Distinctive image features from scale-invariant keypoints. International Journal of Computer Vision, 60 (2004) 91-110

7. Rosten, E., Drummond, T.: Machine learning for high-speed corner detector. In: Computer Conference on Computer Vision (2006) 430-443

8. Smith, S.M., Brady, J.M.: SUSAN - a new approach to low level image processing. International Journal of Computer Vision, 23 (1997) 45-78

9. Trajkovic, M., Hedley, M.: Fast corner detection. Image and Vision Computing, 16 (1998) 75-87

10. Veelaert P.: Constructive fitting and extraction of geometric primitives. CVGIP: Graphical Models and Image Processing, 59 (1997) 233-251

11. Simoncelli, E.P., Farid, H.: Steerable Wedge Filters for Local Orientation Analysis. IEEE Transactions on Image Processing, 5 (1996) 1377-1382

12. Perona, P.: Steerable-scalable kernels for edge detection and junction analysis. In: ECCV, (1992) 3-18 